\title{
CONGENITAL NASOLACRIMAL DUCT OBSTRUCTION IN THE SECOND YEAR OF LIFE: A MULTICENTRE TRIAL OF MANAGEMENT
}

\author{
J. D. H. YOUNG ${ }^{1}$, C. J. MacEWEN ${ }^{1}$ and S. A. OGSTON ${ }^{2}$ \\ Dundee
}

Collaborating surgeons: G. J. Bedford, Dumfries; W. Church, Aberdeen; L. Esakowitz, Paisley; D. Holding, Glasgow; E. G. Kemp, Ayr; C. J. MacEwen, Dundee, G. T. Millar, Edinburgh; J. D. H. Young, Dundee

\begin{abstract}
SUMMARY
We studied spontaneous resolution of congenital nasolacrimal duct obstruction in the second year of life and compared this with the cure rate after probings undertaken between the ages of 11 and 15 months. Of the 111 eyes of 95 patients studied, 26 eyes were included in a randomised prospective comparison of probing with spontaneous resolution. $A$ further 63 eyes followed a similar management plan to the randomised group and are reported as an observational study. Thirty of the 50 eyes followed up without treatment resolved spontaneously before the age of 2 years, of which 24 resolved before 18 months. The overall cure rate for probing was $74 \%$ compared with $60 \%$ for spontaneous resolution. At 15 months of age the randomised study confirmed that probing at 12-14 months is an effective intervention compared with spontaneous resolution $(p=0.04)$. At 24 months of age probing was superior in both randomised and nonrandomised studies, but with increased numbers in the spontaneous resolution groups the difference was no longer statistically significant. Up to 18 months of age the frequency of spontaneous resolution makes delay in probing a viable management option to be discussed with the parents. It will also lead to an overestimate of the cure rate in any study of interventional treatment unless controls are included.
\end{abstract}

Congenital nasolacrimal duct obstruction (CNLDO) is a common problem affecting up to $20 \%$ of all infants ${ }^{1}$ and by far the commonest outcome is spontaneous resolution without surgical intervention. $^{2-5}$ In an observational population-based cohort

From: Departments of ${ }^{1}$ Ophthalmology, and ${ }^{2}$ Epidemiology and Public Health, Ninewells Hospital and Medical School, Dundee, UK.

Correspondence to: Dr J. D. H. Young, Department of Ophthalmology, Ninewells Hospital, Dundee DD1 9SY, UK. study, $96 \%$ of the affected infants were free of symptoms by their first birthday without treatment. ${ }^{1}$ Any treatment proposed must therefore be evaluated against the baseline of this high rate of spontaneous resolution.

On the same basis the natural history of the condition during the second year of life must be known to assess fully the results of any intervention, in particular the standard treatment of probing and syringing the nasolacrimal duct. However, published information on the frequency of spontaneous resolution after 12 months of age is scarce, ${ }^{2,5}$ with that of Nucci et al. ${ }^{6}$ being the most relevant. They reported that in 23 of 29 patients over the age of 12 months the condition resolved within 90 days of commencing 'medical' treatment with sac compression and topical antibiotics.

The primary purpose of the present study was, therefore, to establish the frequency of spontaneous remission in presumed CNLDO persisting into the second year of life: firstly to determine whether it was common enough to be considered as a management option as it is in the first year of life, and secondly to provide a baseline against which any interventional treatments can be assessed. The study was extended to compare spontaneous resolution with the current standard management used by the authors (probing at about age 12 months). The study design was for a prospective controlled randomised trial, with probing at 12-14 months of age compared with no treatment until 24 months. Surgeons at seven centres collaborated in the trial.

\section{METHODS}

All infants presenting with presumed CNLDO during the period of the study, and who were approaching or just after their first birthday, were 
assessed for trial entry. The clinical diagnosis of presumed CNLDO was based on a history of epiphora and/or discharge starting within 3 months of birth, and an abnormal fluorescein dye disappearance test (FDDT). The FDDT was performed in a standard manner and recorded at 5 or 10 minutes. ${ }^{7}$ When present the clinical signs of discharge, a thick tear meniscus, mucocoele and skin excoriation supported the diagnosis, and other conditions such as epiblepharon and chlamydial conjunctivitis were excluded.

Data on the severity of the symptoms were collected at presentation. Parents were asked to grade both the epiphora and discharge, as none, intermittent or constant.

\section{Study Entry}

Patients were divided into four groups as follows.

Group 1. All patients having a mucocoele, or who had persistent conjunctival hyperaemia and discharge except while on antibiotics, were assumed to have a poorer prognosis for spontaneous remission and delay in treatment was considered to be unethical. They were excluded from randomisation, placed in group 1 , and probing advised.

Groups 2 and 3. Parents of those remaining children presenting within the time limits and with no medical contraindication were then given full information on the nature and purpose of the study, including a parents' information sheet approved by Tayside Committee for Medical Ethics. If informed consent was obtained the child was registered by telephone with the study office and a trial number allocated from random number tables (sets of 1-16). Children with odd trial numbers were placed in group 2 and probed between 12 and 14 months. Those with even trial numbers were placed in group 3 and probing delayed until 24 months in the hope of spontaneous remission.

Group 4. All remaining children, including those suitable for the trial, but for whom informed consent was not given, were placed in group 4 and managed according to each clinician's current practice.

Children with a history of previous lacrimal procedures were excluded.

Data were collected prospectively in a defined order on a printed trial form to facilitate standardisation of methods, and of the randomisation process.

\section{Probing}

Probing was carried out with the child under general anaesthetic, and was performed and recorded using an agreed standard protocol. Probing of the nasolacrimal duct was via the upper canaliculus but patency of the lower canaliculus was also confirmed with gentle probing to the sac. The probe wire sizes used were also standardised between the different centres, and patency of the system judged by the identification of diluted fluorescein in the nose or nasopharynx after syringing. The maximum probe size used was 00 (diameter $0.73 \mathrm{~mm}$ approx.). In this paper the term 'probing' is used for the whole procedure, including confirmation of patency by syringing fluorescein.

\section{Follow-up}

At each follow-up visit a standard set of data was collected. Cure was judged on the basis of a complete or near complete remission of symptoms and signs and a normal FDDT. Because of the possibility that spontaneous remission might occur some time after a failed probing, cure from probing was defined as resolution of signs and symptoms within 1 month of the procedure. In practice most cures were identified by parents within a week of the procedure. Three infants were not brought back to clinic and only telephone follow-up was possible.

\section{Obstacles to Randomisation}

Many infants suitable for the trial were not randomised. At this age multiple illnesses in children and their families means there are many missed appointments or delayed probings, and the time limits for trial entry and probing were narrow. We also observed that after parents had read the information sheet and realised there were choices in management many did not wish to agree to the loss of control implicit in the randomisation. The selection of a full 12 months of treatment delay as one arm of the trial may also have inhibited recruitment. The reasons given for non-randomisation are listed in Table I.

\section{Study Population}

A total of 101 completed patient data forms were received but 6 were excluded because of incomplete data or lost follow-up. There were therefore 95 patient records (111 affected eyes) available for analysis. Of these, only 22 patients (26 eyes) were randomised but a further 55 patients (63 eyes) followed a management plan similar to that for the randomised group. This larger non-randomised group provides valid observational data in its own right but is also reported to allow assessment of any selection bias in the randomised groups. In the nonrandomised group the time limits for probing were

Table I. Reasons for non-randomisation

\begin{tabular}{lc}
\hline Reason recorded & Patients \\
\hline Medical decision & 27 \\
Parental decision & 21 \\
Outside time limits & 22 \\
Other reason & 1 \\
Not recorded & 2 \\
Total & 73 \\
\hline
\end{tabular}


relaxed to include patients probed at 11-15 months rather than $12-14$ months as in the randomised group.

\section{RESULTS}

In Table II overall results from the non-randomised and randomised groups are presented in parallel to enable comparison. In the randomised arm of the study probing is statistically superior to spontaneous resolution at 15 months, a difference still evident at 24 months but no longer statistically significant. This pattern is also evident with the larger numbers in the non-randomised groups, but the statistical comparison between them must be interpreted with caution in view of the potential for bias in case selection. If the results of both studies are combined the overall cure rate for probing at $11-15$ months is $74 \%$, compared with $60 \%$ for spontaneous resolution before age 2 years.

The rate at which spontaneous remission occurred during the second year of life in both groups is shown in Fig. 1. In the whole study 30 of the 50 patients $(60 \%)$, observed without treatment, had had a resolution of their CNLDO before their second birthday. We planned a randomised trial to exclude the bias that might result from the parents of the more severely affected children requesting probing. Bias might also have occurred because of the relatively small number of patients in the randomised trial. These points are addressed in Table III, which reports the prevalence of the most severely affected in various groups. However, as there is a subjective element in these classifications and the groups are of different size, these results can only exclude any gross discrepancies in severity between the groups. The incidence of almost constant discharge in the probed groups $(36 \%)$ is greater than that in the delayed treatment groups $(26 \%)$, but the difference is not statistically significant. If the group 1 patients who were probed on medical advice and the randomised patients are excluded, the incidence of almost constant discharge is $26 \%$ (5 of 19) in the probed group and $29 \%$ (10 of 34 ) in the delayed treatment group. We also wished to establish whether the severity at presentation could predict the chance of cure by either probing or spontaneous resolution. Table IV shows that no major association between severity at presentation and cure was demonstrated. However, there was a small group of 5 patients who had epiphora and an abnormal FDDT but whose parents reported no discharge. All 5 showed spontaneous resolution and while the numbers are small the trend approaches significance $(p=0.063$, chi-squared test for trend).

The outcomes in those patients whose treatment failed are shown in Table V. It is of interest that in both groups spontaneous resolution continued to occur, with a further 5 eyes resolving after age 2 years.

The results for the group 1 patients are shown separately in Table VI. These are the patients excluded from randomisation on the assumption

Table II. Summary of the results of both studies

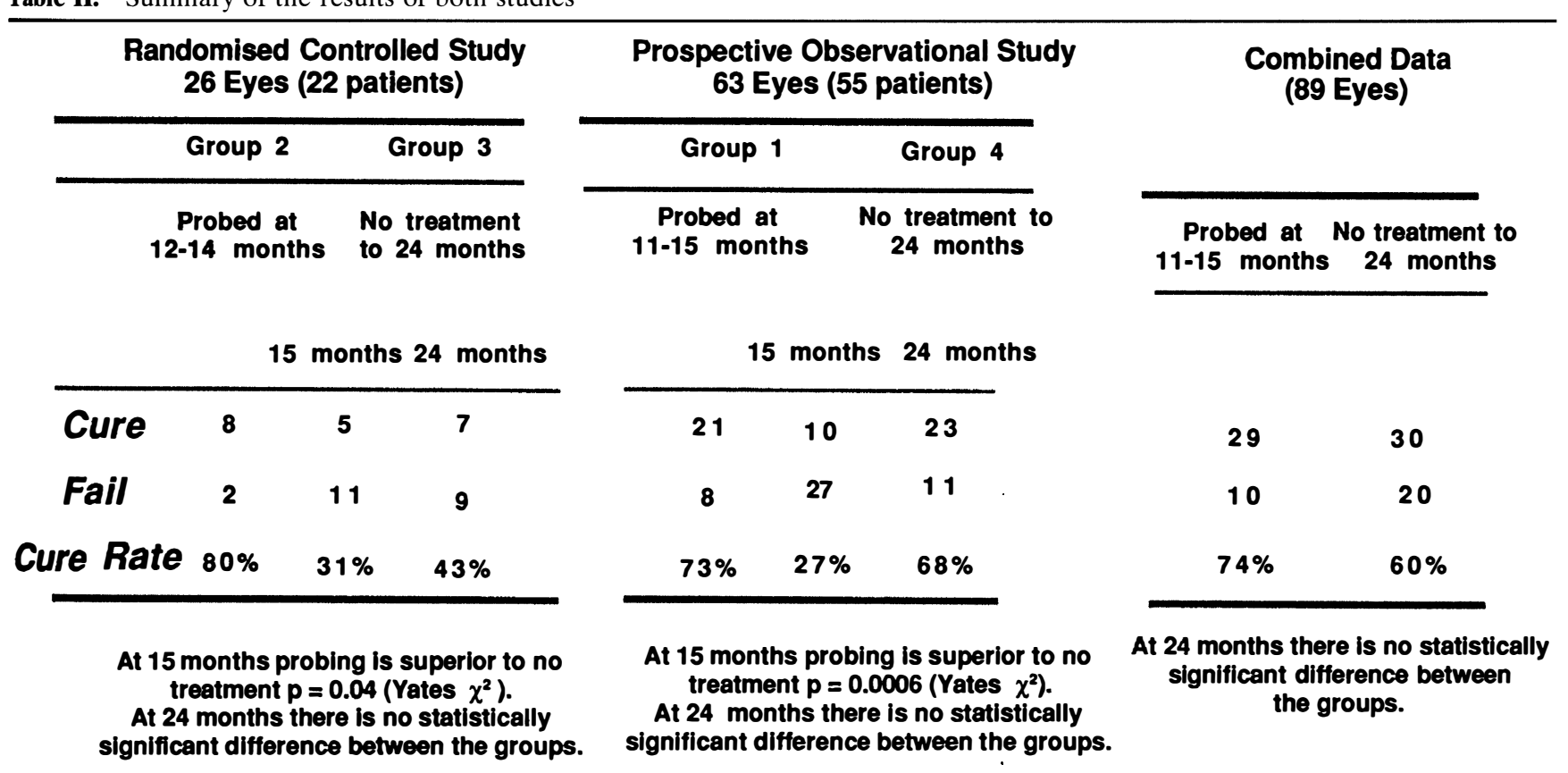

In the non-randomised groups the statistical comparisons must be treated with caution as some bias in case selection cannot be excluded. The figures are reported to show the consistency with results of the randomised study. At 15 months there are three additional patients in the observational study - who were then probed before 24 months. 


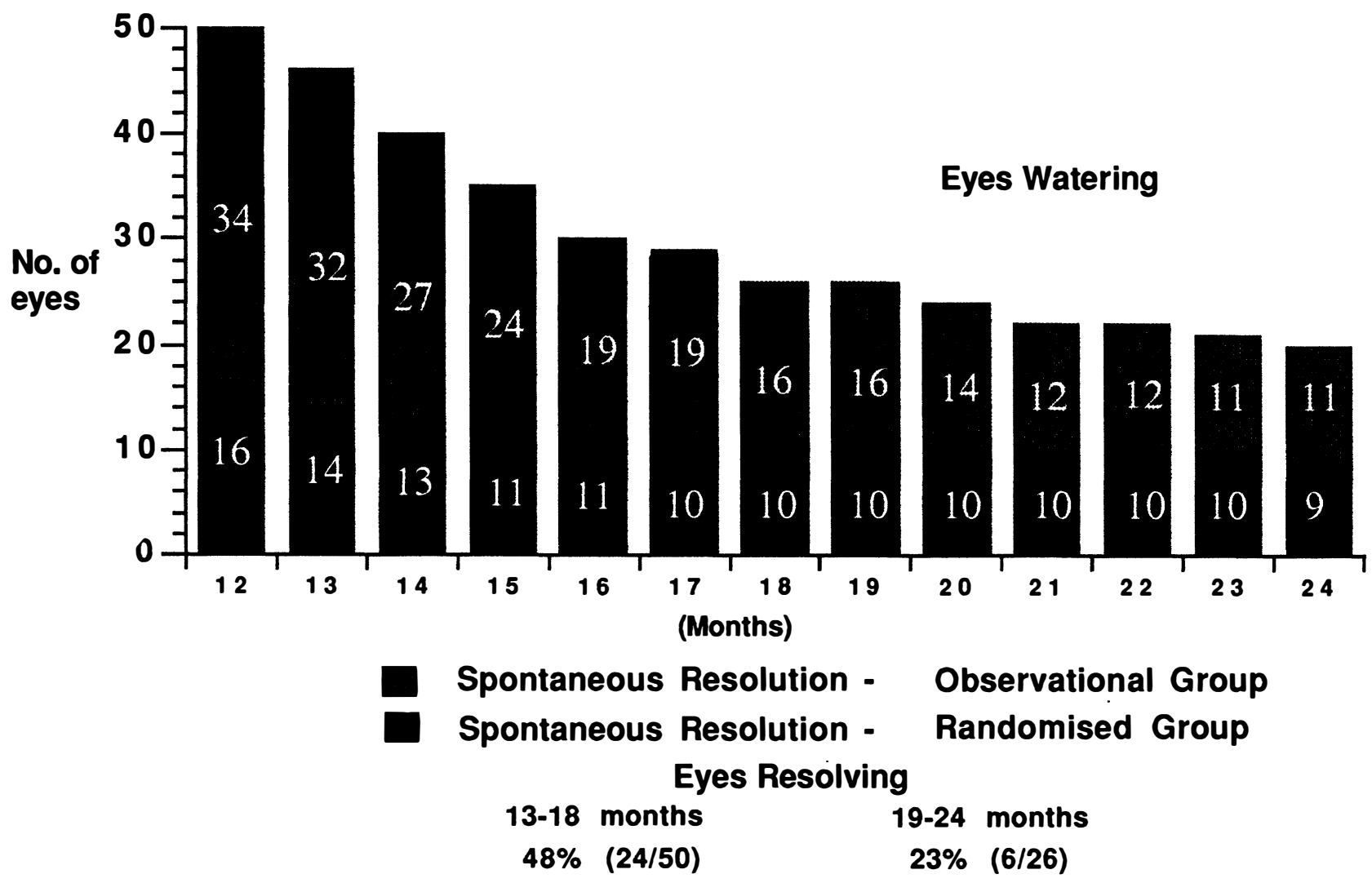

The rate of spontaneous resolution in months $19-24$ was only half that in months $13-18$ and this difference reaches borderline statistical significance.

$$
p=0.06 \text { Yates } \chi^{2}
$$

Fig. 1. Randomised and observational studies: spontaneous resolution.

that with severe symptoms the chances of spontaneous resolution would be less and delay in probing therefore unjustified. In fact the numbers and cure rate at $80 \%$ was exactly the same as that for the randomised patients who were probed, and a further 2 patients showed spontaneous resolution before they could be probed.

Finally some data on the probings in the whole study group are reported (111 eyes of 95 patients). Of the 60 probings undertaken in this study 25 (42\%) were graded as easy with no detectable obstruction to the probe. Of these, 7 cases failed to resolve after the initial probing but 3 then resolved spontaneously before any further treatment. In 20 (33\%) there was a detectable obstruction at the lower end of the nasolacrimal duct (NLD), and in $6(10 \%)$ the

Table III. The severity of symptoms

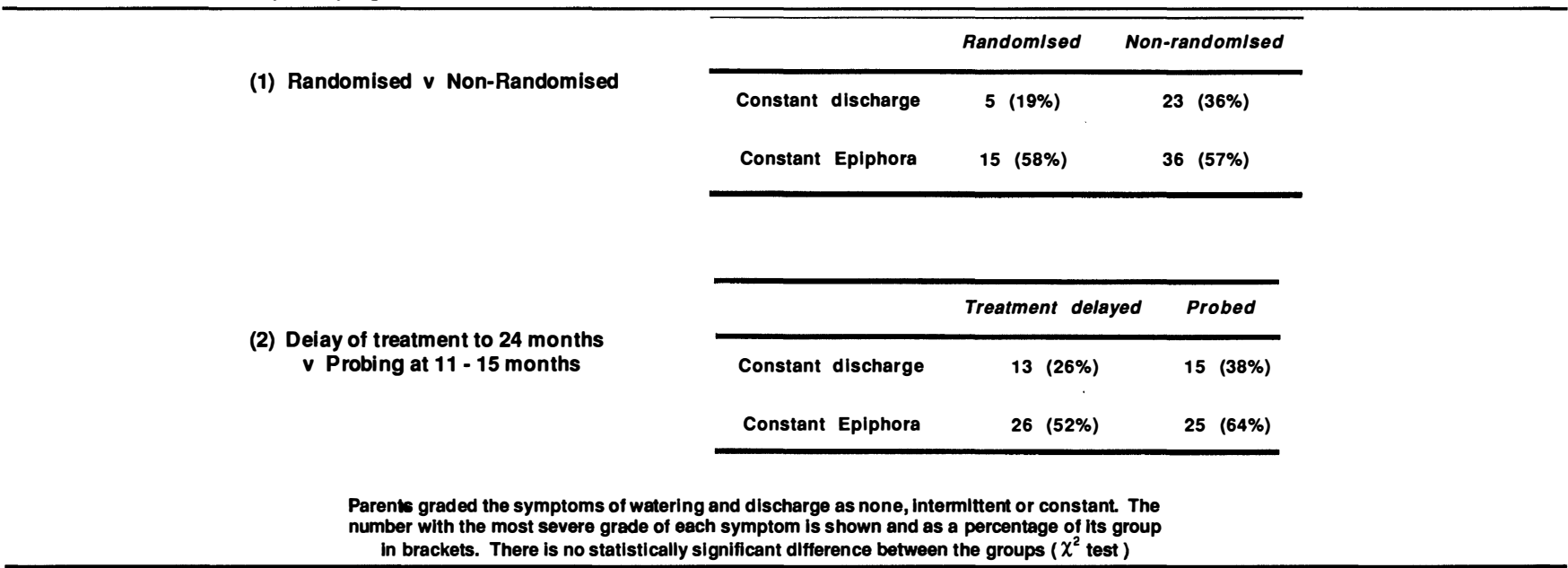


Table IV. Does the severity of symptoms predict the result of treatment?

\begin{tabular}{|c|c|c|c|c|c|c|}
\hline \multicolumn{6}{|c|}{ Probing at 11 - 15 months } & \\
\hline & \multicolumn{2}{|c|}{ Watering } & & \multicolumn{2}{|c|}{ Discharge } & \\
\hline & Cure & Fail & & Cure & Fail & \\
\hline Nil & 1 & 1 & Nil & 1 & 0 & \\
\hline Intermittent & 9 & 3 & Intermittent & 18 & 4 & \\
\hline Constant & 19 & 6 & Constant & 10 & 6 & \\
\hline \multicolumn{6}{|c|}{ Spontaneous resolution by 24 months } & \\
\hline & \multicolumn{2}{|c|}{ Watering } & & \multicolumn{2}{|c|}{ Discharge } & \\
\hline & Cure & Fail & & Cure & Fail & \\
\hline Nil & 1 & 0 & Nil & 5 & $\mathbf{0}$ & \\
\hline Intermittent & 13 & 10 & Intermittent & 19 & 13 & \\
\hline Constant & 16 & 10 & Constant & 6 & 7 & \\
\hline $\begin{array}{l}\text { There was } \\
\text { treatment. } \\
\text { spor }\end{array}$ & $\begin{array}{l}\text { stically } \\
\text { atients } \\
\text { sly. De }\end{array}$ & $\begin{array}{l}\text { nt ass } \\
\text { erlng b } \\
\text { small }\end{array}$ & $\begin{array}{l}\text { tween severity } \\
\text { large in the dela } \\
\text { his is suggestiv }\end{array}$ & $\begin{array}{l}\text { toms ar } \\
\text { atment } \\
\text { nd }\left(\chi^{2}\right.\end{array}$ & $\begin{array}{l}\text { esults of } \\
\text { esolved } \\
\text { 3) }\end{array}$ & \\
\hline
\end{tabular}

obstruction was at the upper end of the NLD. In the remaining 9 eyes $(15 \%)$ severe obstruction to the probe was recorded; 5 of these cases were cured by their initial probing. In 4 patients ( 5 eyes) excluded from the randomised or observational studies, severe punctal stenosis or occlusion was considered to explain the epiphora. Their lacrimal passages were otherwise normal and all were cured by punctal dilation and probing. Bleeding from the punctum was recorded in $12(20 \%)$ of the 60 eyes probed; of these, 8 were cured by the procedure and 4 were not.

\section{DISCUSSION}

The first objective of this study was to determine whether the spontaneous resolution of CNLDO is of clinical significance in the second year of life. With 30 of the 50 patients $(60 \%)$ observed without treatment showing spontaneous resolution by their second birthday this clearly is the case, and parents should be invited to consider delayed treatment as one of the options for management. The data are also very

Table V. Outcome after failure of treatment

\begin{tabular}{|c|c|c|c|}
\hline \multicolumn{4}{|c|}{ Outcome After Failure of Spontaneous Resolution by 24 Months } \\
\hline Outcome & Randomised & Non randomised & Total \\
\hline $\begin{array}{c}\text { Spontaneous } \\
\text { resolution }\end{array}$ & 2 & 3 & 5 \\
\hline Cure after probing & 4 & 2 & 6 \\
\hline Fall after probing & 1 & 2 & 3 \\
\hline $\begin{array}{l}\text { Spontaneous } \\
\text { resolution after } \\
\text { probing }\end{array}$ & 0 & 2 & 2 \\
\hline Lost to follow up & 2 & 2 & 4 \\
\hline Total & 9 & 11 & 20 \\
\hline \multicolumn{4}{|c|}{ Outcome After Failure of Probina at 11 - 15 Months } \\
\hline \multicolumn{2}{|c|}{ Outcome } & \multicolumn{2}{|l|}{ Comments } \\
\hline \multicolumn{2}{|c|}{ Spontaneous resolution } & nonths after probing & 3 \\
\hline \multicolumn{2}{|c|}{ Further procedure } & \multirow{3}{*}{$\begin{array}{l}3 \text { probing, } 1 \text { intubation } \\
\text { After failure recorded }\end{array}$} & 4 \\
\hline Lost to fo & & & 3 \\
\hline Tota & & & 10 \\
\hline
\end{tabular}

suggestive of a decrease in the frequency of spontaneous remission after 18 months of age. Although this difference only reaches borderline statistical significance ( $p=0.06$, Yates' chi-squared), in the absence of alternative data we consider it should be incorporated into the clinical management plan for those children whose parents have elected to defer probing. Should there still be no sign of spontaneous resolution, the age of 18 months appears to be an appropriate time to reconsider probing.

We also observed spontaneous resolution in 7 eyes in the third year of life. The knowledge that spontaneous resolution may still occur after 2 years of age may be relevant to the parents of children with mild symptoms who would prefer to avoid intervention if possible.

Our study has shown that spontaneous resolution remains a common occurrence in the second year of life and this information should be taken into account when assessing the merits of the many treatments that have been proposed for CNLDO. At all ages

Table VI. Group 1 results

\begin{tabular}{|c|c|c|c|}
\hline & \multicolumn{3}{|c|}{$\begin{array}{l}\text { Non-randomised - severe symptoms. } \\
\text { Mucocele or recurrent conjunctivitis with hyperaemia } \\
13 \text { patients (15 eyes) }\end{array}$} \\
\hline & \multicolumn{2}{|c|}{ Probed } & $\begin{array}{l}\text { Spontaneous } \\
\text { Resolution }\end{array}$ \\
\hline & $11-15$ months & $16 \& 17$ months & \\
\hline Cure & 8 & 2 & (14 \& ${ }^{2}{ }^{2}$ months) \\
\hline Fail & 2 & 0 & 1 \\
\hline
\end{tabular}

The cure rate for probing at $11-15$ months is exactly the same as that in the randomised patients who were probed. 
uncontrolled studies of any form of interventional treatment will be likely to overestimate the cure rate. For example, nasolacrimal duct intubation after failed probing has never been evaluated against a control group. The apparent improvement in the success rate obtained by leaving the intubation in situ for 7-12 months ${ }^{8.9}$ may result partly from masked spontaneous remission. However, the present study has illustrated the problems in obtaining control groups in this condition and in some situations the data we have presented may be useful as a guide when analysing reports of interventional treatment.

There may also be occasions when intubation after a failed probing is undertaken unnecessarily. If there is failure of an apparently easy probing, with no resistance to the probe and with patency to fluorescein, then there may be no anatomical obstruction for probing or intubation to cure.

We speculate that in some cases presumed CNLDO may result from poor lacrimal pump function, perhaps due to narrow lacrimal passages in infancy. Three patients in this study showed late spontaneous resolution, after probing graded as easy had failed. In this category of patient we now advise delay until 24 months of age in the hope of spontaneous resolution, before considering intubation of such anatomically patent systems.

The second aim of the study was to reassess probing by comparing its results with spontaneous resolution. The numbers in the randomised study are smaller than intended for the reasons already given. Despite these small numbers some conclusions are possible. At 15 months the cure rate for patients probed at $12-14$ months was $80 \%$ compared with $31 \%$ for no treatment, and this difference is statistically significant - a pattern replicated in the non-randomised group. This provides the first confirmation from a controlled study that probing at 12-14 months is an effective intervention compared with the background rate of spontaneous remission. In both randomised and non-randomised groups probing still appears superior at 24 months, but the increased number of cases with spontaneous resolution in the no-treatment groups means that the difference is not statistically significant.

The apparent advantage of probing at 12-14 months does not mean that it is necessarily the treatment of choice for all patients. Many parents will be more concerned at the prospect of operative intervention and an anaesthetic than the persistent symptoms, and will prefer to delay treatment given a reasonable chance that spontaneous resolution may occur. For most individuals there is no absolute medical indication for either treatment choice and the parents' own views should be decisive.

To make an informed decision the parents need to know the success rates for probing compared with the rate of spontaneous resolution as identified in this study, and to realise that all interventions carry an element of risk. For example, probing a nasolacrimal duct destined for spontaneous resolution might be associated with local trauma inducing canalicular or duct stenosis. ${ }^{10}$ In this study bleeding from the punctum, which might signal such trauma, occurred in $20 \%$ of all probings.

The parents will also wish to know whether delay in treatment carries any risk. This has been suggested as a possibility in the past, either by CNLDO predisposing to severe infections or by chronic infection inducing permanent stenosis of the lacrimal passages. ${ }^{11.12}$ No serious infective complications occurred in this study, or in our study on children in the first year of life. ${ }^{1}$ However, a few cases of orbital cellulitis considered secondary to lacrimal sac infection are cited in the literature and the parents should be advised of this. ${ }^{12.13}$

We consider that there is no valid evidence to confirm the suggestions ${ }^{11.12}$ that delay in probing is detrimental to the cure rate. Katowitz and Welsh ${ }^{12}$ found a drop in the cure rate for probing from $96 \%$ at ages $6-13$ months to $33 \%$ after 2 years, and on this basis they advised probing prior to 13 months of age. This analysis may require revision in view of the high rate of spontaneous resolution we have identified, which will generate an increased incidence of the more severe obstructions in the later-probed groups. In the present study 11 children were probed after age 2 years with a cure rate of $54 \%$. However, at 12 months of age they had been part of a group of 50 infants, 35 of whom eventually showed spontaneous resolution, giving a total cure rate after the probings of $82 \%$ ( $8 \%$ were lost to follow-up after 2 years).

In a birth cohort study $96 \%$ of children originally affected by CNLDO had had spontaneous resolution of the condition by 12 months of age. ${ }^{1}$ Extrapolating this figure on the basis of the frequency of spontaneous resolution found in the present study, less than $2 \%$ of ducts may remain obstructed by age 24 months. If this group could be identified earlier, delay in their treatment could be avoided. Unfortunately we were unable to demonstrate any major correlation between cure rate and severity of symptoms or signs, so there is at present no means of predicting outcome on an individual basis. The only trend that did emerge was for spontaneous resolution in the small group of infants with abnormal FDDT results but whose parents reported only epiphora and not discharge. We suggest probing should be delayed until 24 months for such patients.

In conclusion, we have emphasised the importance of the natural history and parental choice in determining the requirement and timing for any intervention in CNLDO. Some previous studies ${ }^{1-6,14}$ have suggested that the medical advice to parents 
should be to delay probing until after 12 months of age. We consider the evidence from the present study would support a policy of probing at about 12 months of age for those infants with severe signs and symptoms of CNLDO. In less severe cases delay until 18 months is appropriate, during which period there is a $50 \%$ chance of spontaneous resolution. In our view there is no objection to further delay if the parents wish it and this should be specifically advised in infants with epiphora but minimal or no discharge.

We thank the study secretary Mrs V. Brown. The work was supported by a grant from the Speed-Pollock Trust.

Key words: Congenital nasolacrimal duct obstruction, Probing, Natural history.

\section{REFERENCES}

1. MacEwen CJ, Young JDH. Epiphora during the first year of life. Eye 1991;5:596-600.

2. Price HW. Dacryostenosis. J Pediatr 1947;30:302-5.

3. Paul TO. Medical management of congenital nasolacrimal duct obstruction. J Pediatr 1958;22:68-70.

4. Peterson RA, Robb RM. The course of congenital obstruction of the naso-lacrimal duct. J Pediatr Ophthalmol Strabismus 1978;15:246-50.

5. Nelson LB, Calhoun JH, Menduke H. Medical management of congenital nasolacrimal duct obstruction. Ophthalmology 1985;92:1187-90.

6. Nucci $\mathrm{P}$, et al. Conservative management of nasolacrimal duct obstruction. J Pediatr Ophthalmol Strabismus 1989;26:39-43.

7. MacEwen CJ, Young JDH. The fluorescein disappearance test: an evaluation of its use in infants. J Pediatr Ophthalmol Strabismus 1991;28:302-5.

8. Katowitz JA, Welsh MG. Timing of silastic tubing removal after intubation for congenital nasolacrimal duct obstruction. Ophthalmic Plast Reconstr Surg 1989;5:43-8.

9. Al-Hussain H, Nasr AM. Silastic intubation in congenital nasolacrimal duct obstruction. A study of 129 eyes. Ophthalmol Plast Reconstr Surg 1993;9:32-7.

10. Lyon DB, et al. Canalicular stenosis following probing for congenital nasolacrimal duct obstruction. Ophthalmic Surg 1991;22:228-32.

11. Ffooks OO. Dacrocystitis in infancy. Br J Ophthalmol 1962;46:422-34.

12. Katowitz JA, Welsh MG. Timing of initial probing and irrigation in congenital nasolacrimal duct obstruction. Ophthalmology 1987;94:698-705.

13. Noda S, Hayasaka S, Setogawa T. Congenital nasolacrimal duct obstruction in Japanese infants: its incidence and treatment with massage. J Pediatr Ophthalmol Strabismus 1991;28:20-2.

14. El-Mansoury $\mathrm{J}$, et al. Results of late probing for congenital nasolacrimal duct obstruction. Ophthalmology 1986;93:1052-4. 